\title{
Orientation and symmetry control of inverse sphere magnetic nanoarrays by guided self-assembly
}

\author{
Michail E. Kiziroglou, ${ }^{\text {a) }}$ Xiaoli Li, David C. Gonzalez, and Cornelis H. de Groot \\ School of Electronics and Computer Science, University of Southampton, Southampton S017 1BJ, \\ United Kingdom \\ Alexander A. Zhukov and Peter A. J. de Groot \\ School of Physics and Astronomy, University of Southampton, Southampton S017 1BJ, United Kingdom \\ Philip N. Bartlett \\ School of Chemistry, University of Southampton, Southampton SO17 1BJ, United Kingdom
}

(Received 8 March 2006; accepted 22 August 2006; published online 11 December 2006)

\begin{abstract}
Inverse sphere shaped $\mathrm{Ni}$ arrays were fabricated by electrodeposition on $\mathrm{Si}$ through the guided self-assembly of polystyrene latex spheres in $\mathrm{Si} / \mathrm{SiO}_{2}$ patterns. It is shown that the size commensurability of the etched tracks is critical for the long range ordering of the spheres. Moreover, noncommensurate guiding results in the reproducible periodic triangular distortion of the close packed self-assembly. Magnetoresistance measurements on the $\mathrm{Ni}$ arrays were performed showing room temperature anisotropic magnetoresistance of $0.85 \%$. These results are promising for self-assembled patterned storage media and magnetoresistance devices. (C) 2006 American Institute of Physics. [DOI: 10.1063/1.2386936]
\end{abstract}

\section{INTRODUCTION}

Nanopatterned magnetic materials are very attractive for fundamental studies on magnetism and applications such as patterned storage media and spintronic devices. However, the limitations of conventional lithography and the high cost of $e$-beam lithography limits widespread applications in this field. To overcome these restrictions, self-assembly has been widely proposed as an alternative technique. Self-assembly is the autonomous organization of components into patterns or structures without human intervention. ${ }^{1}$ Such structures can be used as templates to transfer the pattern to magnetic materials. A variety of natural systems with self-assembly properties has been used for this purpose including lyotropic liquid crystals, ${ }^{2}$ anodized alumina membranes, ${ }^{3}$ block copolymers, ${ }^{4}$ and close packed arrays of polystyrene latex and silica spheres. ${ }^{5,6}$

While these systems provide well-ordered, short range periodic templates, they lack long range order and the ability to determine the precise position and direction of the periodic structure. In order to address these issues, guiding of the self-assembly has been proposed. This is a hybrid method involving the prepatterning of the substrate using conventional fabrication techniques to assist the self-assembly process. Guided block copolymer self-assembly has been studied showing remarkable guiding effects, ${ }^{7}$ and lithographically defined patterns have been used to aid close packed sphere self-assembly. ${ }^{8-13}$

In comparison with block copolymers, close packed sphere self-assembly techniques are significantly different as they can produce arrays of three-dimensional (3D) magnetic spherical structures in dimensions not accessible by any

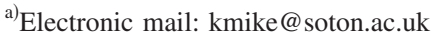

other means. Block copolymer techniques do not allow control of the shape of the nanostructures. ${ }^{14}$ Furthermore, the pattern transfer process is considerably simpler for spheres, as it does not involve any intermediate materials. Finally, while both techniques can be used to fabricate isolated magnetic dots or pillars, ${ }^{14-17}$ nanosphere lithography has the unique advantage of creating inverse sphere structures. ${ }^{5}$

In addition to the aforementioned differences, the elastic response of block copolymers to patterned substrates results in a different guiding behavior compared with that of the close packed spheres. While block copolymers adjust their period to match the width of the pattern, ${ }^{7}$ spheres have an almost inelastic behavior. Therefore, guidance has to be studied separately for these two techniques.

The combination of lithographic patterns and sphere self-assembly has been studied, and the importance of dimensional commensurability has been recognized. ${ }^{18,19}$ However, no close packed submicrometer sphere arrangements have been achieved and no systematic variation of the sphere arrangements with commensurability was detected. Furthermore, such hybrid structures have not yet been exploited for the fabrication of nanoscale magnetic structures.

In previous papers, we have studied the magnetic properties of self-assembled 3D inverse sphere magnetic arrays. ${ }^{17,20}$ We have also reported initial results on guided inverse sphere arrays highlighting the significance of the shape of the guiding patterns. ${ }^{21}$ Here, a detailed analysis for the guided self-assembly of inverse sphere magnetic monolayers is presented. The response of close packed sphere selfassembly to guiding stripes with different widths is studied. Guided inverse sphere magnetic monolayers directly on $\mathrm{Si}$ are fabricated and magnetoresistance measurements are performed. 
(a)

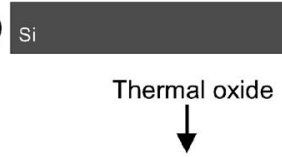

(b)

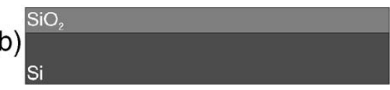

Photoresist spin

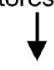

(c)

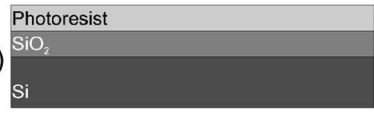

Photoresist developing

(d)
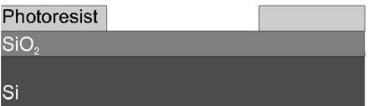

Wet Etch and Resist Strip

(e)

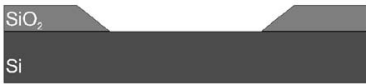

(g)

(f)

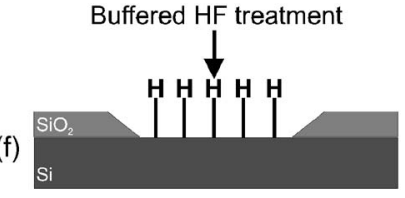

(h)

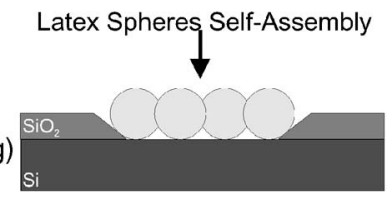

Buffered HF treatment

(h)

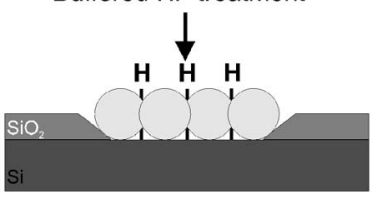

Ni Electrodeposition

(i)

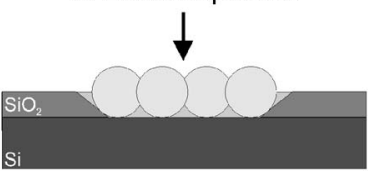

Spheres Dissolution

(j)

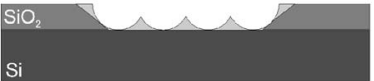

FIG. 1. Process flow for the guided self-assembly of inverse sphere magnetic arrays.

\section{EXPERIMENTAL METHODS}

A description of the fabrication process is illustrated in Fig. 1. $N$-type (100) single-side polished silicon wafers with resistivity of $0.01-0.02 \Omega \mathrm{cm}$ were used as substrates [Fig. 1(a)]. A $250 \mathrm{~nm}$ thick layer of $\mathrm{SiO}_{2}$ was thermally grown on the front side [Fig. 1(b)] and photoresist was spun on top [Fig. 1(c)]. Guiding stripes with thicknesses ranging from 1.1 to $12 \mu \mathrm{m}$ were transferred to the photoresist from an optical mask using a Nikon NSR-2005/i9C step and repeat system [Fig. 1(d)]. Subsequently, the wafers were immersed in a 20:1 buffered hydrogen fluoride (BHF) solution which etched the oxide and exposed the $\mathrm{Si}$ at the selected areas, followed by a fuming nitric acid clean to remove the photoresist [Fig. 1(e)]. At that point the wafers were sawed in pieces and cleaned using an aqueous mixture of hydrogen peroxide and ammonium hydroxide and a mixture of hydrogen peroxide and $\mathrm{HCl}$ (Radio Corporation of America, RCA clean).

A 20:1 BHF dip followed, leaving the $\mathrm{Si}$ surface $\mathrm{H}$ terminated and hence, hydrophobic. Shortly after, $500 \mathrm{~nm}$ diameter latex spheres were self-assembled on the substrates by the slow evaporation of a colloidal water suspension containing 1 wt. \% of latex spheres. In most of the cases, trapping of the spheres into the morphological patterns of the substrate resulted in selective sphere self-assembly on Si.

Electrodeposition through the self-assembly of the latex spheres was used to grow inverse sphere $\mathrm{Ni}$ arrays directly on $\mathrm{Si}$ without a back contact. Just before electrodeposition, another 20:1 BHF dip was necessary to remove the native oxide and leave the Si surface H terminated [Figs. 1(h) and 1(i)]. An Autolab AUT72032 potentiostat three electrode sys-

tem with a Pt counter electrode and a saturated calomel reference electrode (SCE) was used. The deposition potential was $-1.1 \mathrm{~V}$ (against the SCE). A pulse of $-1.5 \mathrm{~V}$ was applied for $0.2 \mathrm{~s}$ just before the deposition stage to form a uniform $\mathrm{Ni}$ nucleation on $\mathrm{Si}$ which led to smoother deposition. ${ }^{22}$ The insulating $\mathrm{SiO}_{2}$ forced electrodeposition to take place selectively on the Si patterns. The thickness of the Ni films was controlled by measuring the charge during electrodeposition. This control process was calibrated against vibrating sample magnetometry measurements on continuous films to take into account hydrogen evolution effects at the cathode during electrodeposition. The latex spheres were dissolved using tetrahydrofuran $\left(\mathrm{C}_{4} \mathrm{H}_{8} \mathrm{O}\right)$, leaving selfassembled inverse sphere Ni arrays on the Si patterns [Fig. $1(\mathrm{j})$ ]. The resulting structures were characterized using scanning electron microscopy (SEM) before and after the electrodeposition stage.

For magnetoresistance measurements, a single layer inverse sphere Ni array was prepared. The Ni thickness was $500 \mathrm{~nm}$. Dissolution of the spheres was omitted to prevent oxidation of the fine $\mathrm{Ni}$ structure on the inner side of the array. Due to the relatively large Ni thickness, surface oxidation was not expected to affect significantly the magnetic properties of the array. Ag pads were defined to allow for electrical measurements. Room temperature magnetoresistance measurements were performed using a square modulated current with frequency of $68 \mathrm{~Hz}$ and a lock-in detection technique. The amplitude of the current was $1 \mu \mathrm{A}$ for measurements with the magnetic field parallel and $10 \mu \mathrm{A}$ for those with the magnetic field perpendicular to the current direction.

\section{RESULTS AND DISCUSSION}

\section{A. Commensurability of guiding patterns}

When a colloidal solution of latex spheres is left to evaporate on a surface, strong attractive capillary forces among the spheres tend to maximize the density of the remaining structure. ${ }^{23}$ Since the maximum sphere density is achieved by close packing, ${ }^{24}$ this mechanism leads to selfassembled cubic or hexagonal close packed multilayers. By adjusting the amount of the colloidal solution, close packed monolayers can also be fabricated. In this manner, selfassembly monolayers of latex spheres with no guidance on $\mathrm{SiO}_{2}$ were prepared as shown in Fig. 2. These structures typically have a uniform orientation for lengths more than $10 \mu \mathrm{m}$. However, the unrestricted deposition area permits the spheres to shift and create fractures.

Self-assembly monolayers of latex spheres guided by 12 and $6 \mu \mathrm{m}$ stripes are shown in Figs. 3(a) and 3(b), respectively. The sphere deposition technique was the same as that used in the case of Fig. 2. Improvement of the self-assembly is observed with one discrete defect in an area of 10 $\times 12 \mu \mathrm{m}$. One of the axes of the hexagonal structures is aligned with the edges of the guiding structures, demonstrating the ability to predetermine the orientation of the self-assembly.

Furthermore, Fig. 3 shows selective sphere deposition on the hydrophobic Si surface rather than on the hydrophilic 


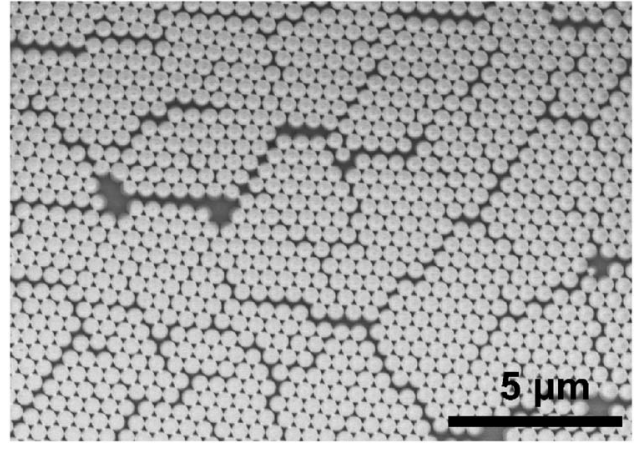

FIG. 2. SEM image of self-assembled latex spheres without guiding. The arrangement is hexagonal and has a uniform orientation, but severe fractioning occurs.

$\mathrm{SiO}_{2}$. This result contradicts studies reported in the literature showing strong preference for deposition on hydrophilic surfaces. ${ }^{25}$ It suggests the domination of the sphere trapping mechanism in the morphological patterns ${ }^{18}$ over hydrophobic/hydrophilic effects. It must be noted, however, that in cases where large amounts of colloidal suspension were used, indiscriminate self-assembly of latex spheres throughout the substrate was observed. These extra spheres on the oxide do not affect the Ni deposition and they are removed by the solvent which removes the spheres from the Ni film.

It is apparent from the left edge of the pattern in Fig. 3 that the self-assembly does not abut against the sides of the guiding stripe. This is because the size of the stripe is not commensurate with the diameter of the spheres. After the electrodeposition step, these nonpatterned spaces would result in continuous magnetic stripes at the sides of the inverse sphere array. Such imperfections would not always be desirable. More importantly, in some cases this mismatch results in low quality guiding. The sphere arrays can glide in the

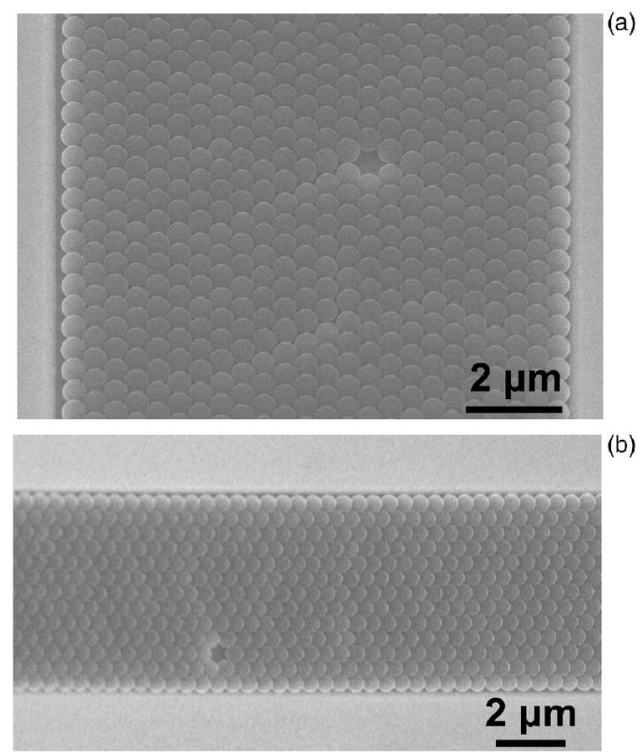

FIG. 3. SEM image of self-assembled latex spheres guided by a $12 \mu \mathrm{m}$ (a) and a $6 \mu \mathrm{m}$ (b) wide $\mathrm{Si} / \mathrm{SiO}_{2}$ stripe. The close packing of the spheres is aligned to the guiding edges. The missing sphere represents the state-of-theart defect rate of our self-assembly technique. (a)

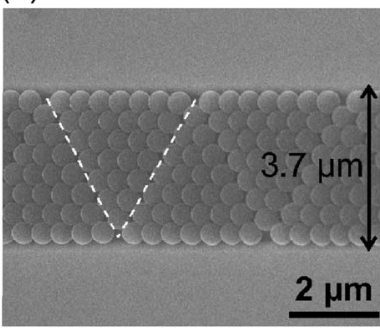

(b)

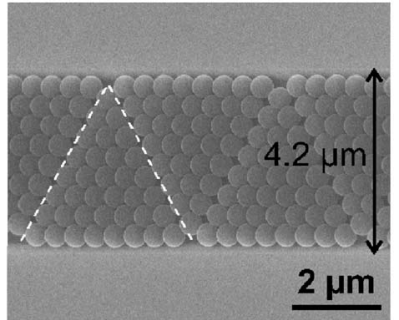

(c)

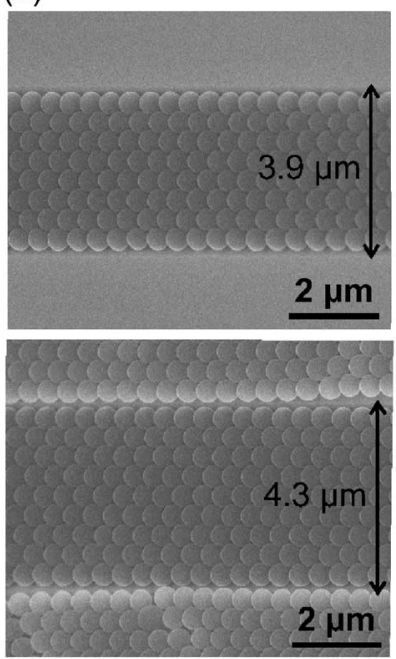

(d)

FIG. 4. In (a), an array of seven rows of spheres is guided by a $3.7 \mu \mathrm{m}$ wide stripe. This excessive width allows for a natural, triangular distortion of the array which increases the sphere density. In (b), an array of eight rows of spheres is guided by a $3.9 \mu \mathrm{m}$ wide stripe. The dimensional match results in a perfectly guided sphere array. Similarly, in (c) an array of eight rows of spheres is guided by a $4.2 \mu \mathrm{m}$ wide stripe resulting in a distorted array. In (d) an array of nine rows of spheres is guided by a $4.3 \mu \mathrm{m}$ wide stripe resulting in perfect guidance. Note that in (d) the sphere self-assembly is expanded to the $\mathrm{SiO}_{2}$ region indicating that the spheres deposition process is not always selective.

patterns deviating from the desired orientation and create fractures. These possibilities make it difficult to achieve good quality structures in noncommensurate cases.

In order to study the dependence of the self-assembly process on the size of the guiding patterns, stripes with various widths were fabricated. In Fig. 4(a), a stripe with a width of $3.7 \mu \mathrm{m}$ is used to guide the sphere self-assembly. The stripe is too big for a sphere array of seven rows and too small for eight rows. The demand of maximum density imposes a structure of seven rows with trigonal symmetrical fractures. Such a structure embodies two levels of selfassembly. One of close packed spheres at the sphere level $(500 \mathrm{~nm})$ and one of equilateral triangles at the grain level (seven spheres a side or $3.5 \mu \mathrm{m}$ ). In Fig. 4(b), a stripe with a width of $3.9 \mu \mathrm{m}$ is used. This size is commensurate with a close packed sphere array of eight rows and hence it works as a perfect guide. Similarly, in Fig. 4(c), a stripe with a width of $4.2 \mu \mathrm{m}$ is used. The stripe is too big for an array of eight rows and too small for nine rows. The demand of maximum density imposes a dual self-assembly structure of eight rows with trigonal symmetrical fractures. The equilateral triangles are eight spheres a side or $4 \mu \mathrm{m}$. Lastly, in Fig. 4(d), a stripe with a width of $4.3 \mu \mathrm{m}$ is used. This size is commensurate with a close packed sphere array of nine rows and perfect guiding is achieved. Note that in this case, spheres are observed on the $\mathrm{SiO}_{2}$ too as a result of the large amount of colloidal suspension used. The guidance of the pattern does even extend onto the $\mathrm{SiO}_{2}$ as the spheres are twice the height of the $\mathrm{SiO}_{2}$ thickness.

The above results on incommensurate guiding are very different from those reported for block copolymers. ${ }^{7}$ The 


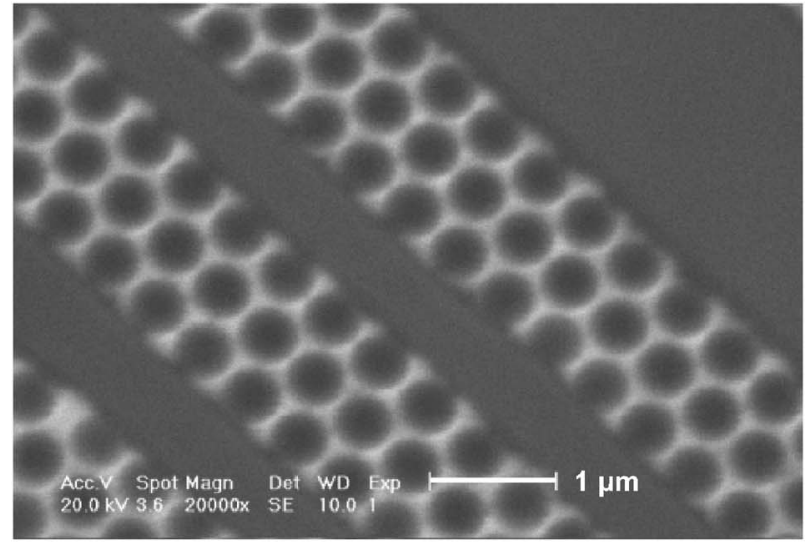

(a)

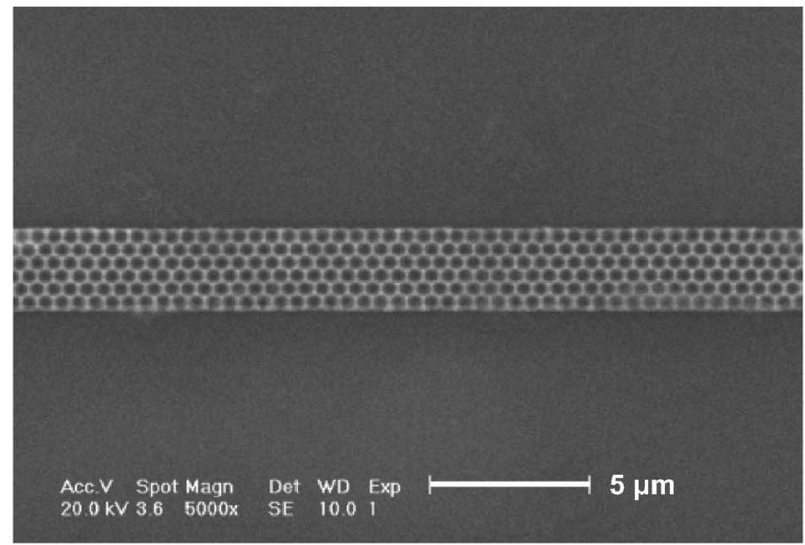

FIG. 5. SEM image of self-assembled inverse sphere Ni arrays guided by (a) $1.3 \mu \mathrm{m} \mathrm{Si} / \mathrm{SiO}_{2}$ and (b) $2.6 \mu \mathrm{m}$ stripes. The uniform, black area is $\mathrm{SiO}_{2}$ while the honeycomblike, white structure is the surface of the Ni. The holes in the array correspond to the spherical $\mathrm{Ni}$ cavities with the $\mathrm{Si}$ bottom appearing black at the centers.

large elasticity of the latter allows deformation to fit the guided pattern. In contrast, the hard nature of latex spheres imposes direct control of the sphere packing arrangement by the size of the guiding patterns. The period of the structures remains constant as predetermined by the size of the spheres used.

These results demonstrate the ability to fabricate perfectly guided sphere self-assembly arrays by tailoring the size of the guiding structure. Moreover, on-demand dual (close packing crystallization and triangular fractioning) selfassembly structures can be constructed. The number of spheres per triangle is controlled by the size of the stripe. Although noncommensurability complicates guidance and increases the sensitivity of the fabrication process the resulting structures are very promising for photonic crystal applications. ${ }^{26,27}$

\section{B. Magnetoresistance in self-assembled inverse sphere Ni arrays}

Commensurately guided close packed sphere arrays as described in Sec. III A were used to fabricate inverse sphere magnetic arrays on $\mathrm{Si}$, as shown in Fig. 5. The array thickness was $250 \mathrm{~nm}$ (half the diameter of the spheres) to allow observation of the inverse sphere structure. Ni electrodeposition occurs only on $\mathrm{Si}$ and not on the insulating $\mathrm{SiO}_{2}$, resulting in the confinement of the magnetic arrays exclusively

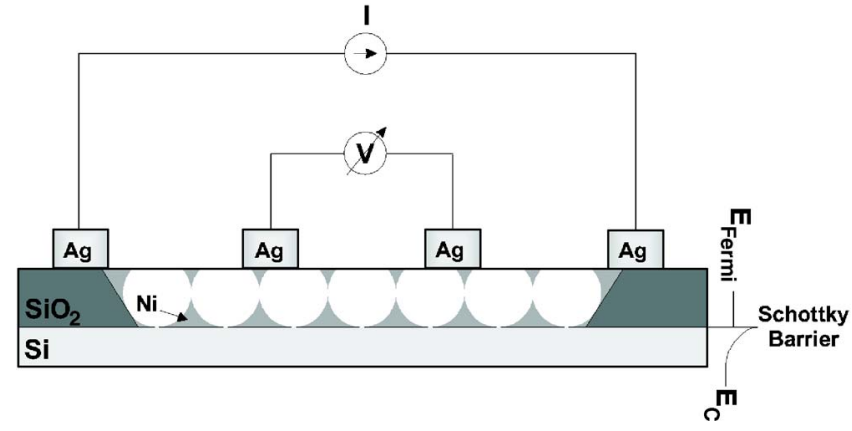

FIG. 6. Four-probe measurements on guided self-assembly of inverse sphere $\mathrm{Ni}$ arrays on Si. The size of the spherical voids is not in scale.

to the $\mathrm{Si}$ patterns. The sphere template pattern has been transferred to the Ni layer without defects. In Fig. 5(a), Ni arrays guided by $1.3 \mu \mathrm{m}$ wide stripes are shown. Good commensurability prevents the formation of uniform Ni lines at the sides of the array. In Fig. 5(b), a Ni array guided by a $2.6 \mu \mathrm{m}$ wide stripe is shown. The guiding stripe is slightly wider than the array resulting in very thin $\mathrm{Ni}$ lines at the edges. This result demonstrates the ability of on-demand bordering of the inverse sphere magnetic arrays.

Predetermination of the position of the array allowed Ag pads to be defined and four-probe magnetoresistance measurements to be performed. A description of the measurement setup is shown in Fig. 6. In such setups, leakage currents flowing through the semiconducting substrate would suppress the observed magnetoresistance effects. For plain magnetic layers with very small Ohmic resistance the substrate leakage current is negligible and magnetoresistance measurements can be performed. ${ }^{28}$ However, for patterned magnetic layers such as the inverse sphere magnetic antisphere arrays shown in Fig. 5 the resistance is substantially higher and suppression of leakages becomes paramount. This can be achieved by exploiting the excellent Schottky barrier at the electrodeposited $\mathrm{Ni}-\mathrm{Si}$ interface. ${ }^{22}$

Magnetoresistance measurements were performed at room temperature on a single layer inverse sphere rectangular Ni array with in-plane dimensions of $230 \times 10 \mu \mathrm{m}$ and height of $500 \mathrm{~nm}$. The net current flow was parallel to the long dimension of the rectangle. The resistance of the array at remanence was $R(0)=12.2 \Omega$. In-plane measurements with the magnetic field parallel to the current are shown in Fig. 7(a). The resistance is at its maximum when the magnetic moments in the array are parallel to the current flow. During the magnetization reversal process, the magnetic moment turns perpendicular to the current flow resulting in lower resistance. The two distinct minima at -8 and $+8 \mathrm{mT}$ indicate some hysteresis in the array. Figure 7(b) shows magnetoresistance measurements with the in-plane magnetic field perpendicular to the current. The resistance is at its minimum when the magnetic moments in the array are aligned perpendicular to the current flow by the magnetic field. No significant hysteresis is observed indicating a reversible rotation. The maximum resistance for this case is observed when no magnetic field is applied.

From Fig. 7, the anisotropic magnetoresistance (AMR) of the array defined as 

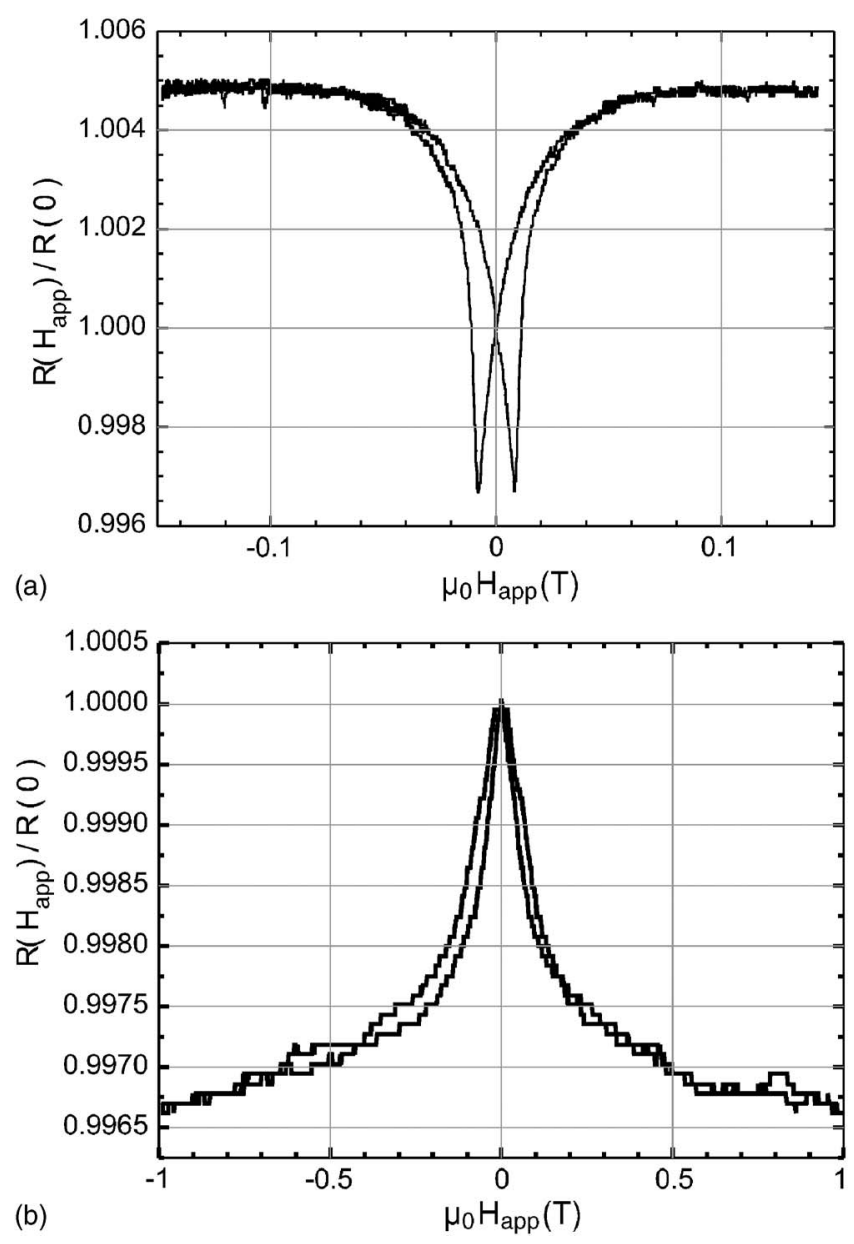

FIG. 7. Room temperature magnetoresistance measurements at guided selfassembly of inverse sphere $\mathrm{Ni}$ arrays on $\mathrm{Si}$ for in-plane magnetic fields, (a) parallel and (b) perpendicular to the current flow.

$$
\mathrm{AMR}=2 \frac{R_{\|}-R_{\perp}}{R_{\|}+R_{\perp}}
$$

can be calculated, where $R_{\|}$and $R_{\perp}$ are the saturation resistances for applied magnetic field parallel and perpendicular to the current flow, respectively. The AMR ratio is found to be $0.85 \%$. Similar results have been observed in CoFe antidot arrays fabricated by anodized alumina techniques but with weaker AMR effects. ${ }^{29}$ This can be attributed to the unique geometry of the inverse sphere arrays which causes interesting magnetization phenomena. ${ }^{30,31}$

It is noted that smaller resistance variation is observed for magnetization reversal in the perpendicular direction. This difference could be attributed to the three-dimensional magnetization pattern of the zero-field state outweighing the easy axis preference. However, closer examination of the magnetization reversal mechanisms in such structures is required for a full explanation of this effect. More detailed studies on the magnetoresistance of these arrays should take into account that the current is not exactly parallel or perpendicular to the magnetization as it follows the inverse spherical geometry.

\section{CONCLUSIONS}

In this paper, a simple, fast, and cost effective guiding technique for orientation and symmetry control of inverse sphere magnetic nanoarrays has been presented. By tailoring the dimensions of the guiding patterns, switching between perfect and triangularly distorted close packed self-assembly symmetries is possible. Higher AMR values than those reported for similar structures are observed. These results are promising for further micromagnetic studies and applications such as patterned storage media and magnetoresistance devices. Our fabrication technique could also be applied to nanoscale self-assembly arrays with customized symmetries for photonic crystals.

\section{ACKNOWLEDGMENTS}

This work was supported by EPSRC, the European Union (SINANO), and Innos Ltd who provided access to clean room processing.

${ }^{1}$ G. M. Whitesides and B. Grzybowski, Science 295, 2418 (2002).

${ }^{2}$ P. N. Bartlett, P. N. Birkin, M. A. Ghanem, P. de Groot, and M. Sawicki, J. Electrochem. Soc. 148, C119 (2001).

${ }^{3}$ J. C. Hulteen and C. R. Martin, J. Mater. Chem. 7, 1075 (1997).

${ }^{4}$ T. Thurn-Albrecht et al., Science 290, 2126 (2000).

${ }^{5}$ P. N. Bartlett, P. R. Birkin, and M. A. Ghanem, Chem. Commun. (Cambridge) 1671 (2000).

${ }^{6}$ L. Xu, W. L. Zhou, C. Frommen, R. H. Baughman, A. A. Zakhidov, L. Malkinski, J. Q. Wang, and J. B. Wiley, Chem. Commun. (Cambridge) 997 (2000).

${ }^{7}$ J. Cheng, C. Ross, and A. Mayes, Nat. Mater. 3, 823 (2004).

${ }^{8}$ S. M. Yang and G. A. Ozin, Chem. Commun. (Cambridge) 2507 (2000).

${ }^{9}$ D. Wang and H. Mohwald, J. Mater. Chem. 14, 459 (2004).

${ }^{10}$ P. Ferrand, M. Egen, B. Griesebock, J. Ahopelto, M. Muller, R. Zentel, S. G. Romanov, and C. M. Sotomayor, Appl. Phys. Lett. 81, 2689 (2002).

${ }^{11}$ J. P. Hoogenboom, C. Retif, E. de Bres, M. van de Boer, A. K. van Langen-Suurling, J. Romijn, and A. van Blaaderen, Nano Lett. 4, 205 (2004).

${ }^{12}$ Y. H. Ye, S. Badilescu, V. V. Truong, P. Rochon, and A. Natansohn, Appl. Phys. Lett. 79, 872 (2001).

${ }^{13}$ J. Zhang et al., Appl. Phys. Lett. 81, 3176 (2001).

${ }^{14}$ J. Y. Cheng, C. A. Ross, V. Z.-H. Chan, E. L. Thomas, R. G. H. Lammertink, and G. J. Vancso, Adv. Mater. (Weinheim, Ger.) 13, 1174 (2001).

${ }^{15}$ S. M. Weekes, F. Y. Ogrin, and W. A. Murray, Langmuir 20, 11208 (2004).

${ }^{16}$ M. E. Abdelsalam, P. N. Bartlett, J. J. Baumberg, and S. Coyle, Adv. Mater. (Weinheim, Ger.) 16, 90 (2004).

${ }^{17}$ A. Zhukov, M. Ghanem, A. Goncharov, P. de Groot, I. El-Hallag, P. Bartlett, R. Boardman, and H. Fangohr, J. Magn. Magn. Mater. 272-276, 1621 (2004).

${ }^{18}$ Y. Yin, Y. Lu, B. Gates, and Y. Xia, J. Am. Chem. Soc. 123, 8718 (2001).

${ }^{19}$ R. K. Golding, P. C. Lewis, E. Kumacheva, M. Allard, and E. H. Sargent, Langmuir 20, 1414 (2004).

${ }^{20}$ A. A. Zhukov, A. V. Goncharov, P. A. J. de Groot, P. N. Bartlett, and M. A. Ghanem, J. Appl. Phys. 93, 7322 (2003).

${ }^{21}$ A. A. Zhukov et al., IEEE Trans. Magn. 41, 3598 (2005).

${ }^{22}$ M. E. Kiziroglou, A. A. Zhukov, M. Abdelsalam, X. Li, P. A. J. de Groot, P. N. Bartlett, and C. H. de Groot, IEEE Trans. Magn. 41, 2639 (2005).

${ }^{23}$ N. D. Denkov, O. D. Velev, P. A. Kralchevsky, I. B. Ivanov, J. H. Yoshimura, and K. Nagayamat, Langmuir 8, 3183 (1992).

${ }^{24}$ J. H. Conway and N. J. A. Sloane, Sphere Packings, Lattices and Groups (Springer-Verlag, Berlin, 1988).

${ }^{25}$ C. A. Fustin, G. Glasser, H. W. Spiess, and U. Jonas, Langmuir 20, 9114 (2004).

${ }^{26}$ R. Wilson, T. J. Karle, I. Moerman, and T. F. Krauss, J. Opt. A, Pure Appl. Opt. 5, S76 (2003).

${ }^{27}$ J. D. Joannopoulos, P. R. Villeneuve, and S. Fan, Nature (London) 386, 143 (1997).

${ }^{28}$ A. Pasa and W. Schwarzacher, Phys. Status Solidi A 173, 73 (1999).

${ }^{29}$ F. J. Castano, K. Nielsch, C. A. Ross, J. W. A. Robinson, and R. Krishnan, Appl. Phys. Lett. 85, 2872 (2004).

${ }^{30}$ A. A. Zhukov et al., Appl. Phys. Lett. 88, 062511 (2006).

${ }^{31}$ M. Tanaka, E. Saitoh, H. Miyajima, and T. Yamaoka, J. Magn. Magn. Mater. 282, 22 (2004) 